\title{
The First Triangular Representation of The Symmetric Groups over a field $\mathrm{K}$ of characteristic pdivides ( $\mathrm{n}-2)$
}

\author{
Eyad M.A.Al-Aamily ${ }^{1}$,AliAbdulsahib M.Al-Butaihi ${ }^{2}$,Abid Ali H.Al-Ta'ai ${ }^{3}$ \\ Department of Mathematics, College of Sciences, Al-Mustansiriyah University,Baghdad, Iraq \\ science@uomustansiriyah.edu.iq \\ Department of Mathematics and computer applications, College of Sciences, Al- \\ NahrianUniversity,Baghdad,Iraq \\ alnahrain sci@hotmail.com \\ Department of Mathematics,College of Sciences, Al-MustansiriyahUniversity,Baghdad, Iraq \\ science@uomustansiriyah.edu.iq
}

\begin{abstract}
In this paper we will study the new type of triangular representations of the symmetric groups which is called the first triangular representations of the symmetric groups over a field $\mathrm{K}$ of characteristic pdivides(n-2).
\end{abstract}

Keywords: symmetric group; group algebra $\mathrm{KS}_{\mathrm{n}}, \mathrm{KS}_{\mathrm{n}}$-module;Specht module ;exact sequence .

\section{Academic Discipline And Sub-Disciplines}

Philosophy of Mathematics,Algebra,Grouptheory,Group representation.

\section{SUBJECT CLASSIFICATION: $20 \mathrm{C} 30$}

\section{TYPE (METHOD/APPROACH)}

Pure Mathematics.

\section{INTRODUCTION}

When Prof. W. Specht was a student under the supervision of Prof. I. Schur, he began investigating representation theory of the symmetric group .During that time it was well known that standard Young tableaux of a given partition $\lambda$ of a positive integer $\mathrm{n}$ form a basis of an ordinary irreducible representation space of $\mathbf{S}_{\mathbf{n}}$. The problem that W.Specht was facing in his investigating in that time is that the symmetric group acts in a natural way on tableaux, but the result of the application of a permutation to a standard tableau can be a nonstandard tableau, and it is by no means clear how a nonstandard tableau can be written as a linear combination of standard ones. For this reason W. Specht introduced in 1935 polynomials corresponding to the tableaux ( known nowadays as Specht polynomials), and it is obvious how a given polynomial can be written as a linear combination of other polynomials (see [1] ).

In 1971 Peel introduced the $r^{\text {th }}$ Hook representations which deals with the partitions $\lambda=\left(n-r, 1^{r}\right) ; r \geq 1$.[4]

In 2016 we introduce in our paper[5] new representations of the symmetric groups we call them the triangular representations of the symmetric groups and we study the first of them which we call it the first triangular representation of the symmetric groups when $p$ divides $(n-1)$.and follow it by the paper[6] which it is study the first triangular representation of the symmetric groups over a field $\mathbf{K}$ of characteristic $p=0$.

Throughout this paper let $\mathbf{K}$ be a field of characteristic $p$ which is divide $(n-2)$, and $x_{1}, x_{2}, \ldots, x_{n}$ be linearly independent commuting variables over $\mathbf{K}$.

\section{PRIEMINERIES}

\section{Definition 2.1:}

Let $\mathbf{S}_{\mathbf{n}}$ be the set of all permutations $\tau$ on the set $\left\{\mathrm{x}_{1}, \mathrm{x}_{2}, \ldots, \mathrm{x}_{\mathrm{n}}\right\}$ and $\mathbf{K}\left[\mathrm{x}_{1}, \mathrm{x}_{2}, \ldots, \mathrm{x}_{\mathrm{n}}\right]$ be the ring of polynomials in $\mathrm{x}_{1}, \mathrm{x}_{2}, \ldots, \mathrm{x}_{\mathrm{n}}$ with coefficients in $\mathbf{K}$. Then each permutation $\tau \in \mathbf{S}_{\mathbf{n}}$ can be regarded as a bijective function from $\mathbf{K}\left[\mathrm{x}_{1}, \mathrm{x}_{2}, \ldots, \mathrm{x}_{\mathrm{n}}\right]$ onto $\mathbf{K}\left[\mathrm{x}_{1}, \mathrm{x}_{2}, \ldots, \mathrm{x}_{\mathrm{n}}\right]$ defined by $\left(\mathrm{f}\left(\mathrm{x}_{1}, \mathrm{x}_{2}, \ldots, \mathrm{x}_{\mathrm{n}}\right)\right)=\mathrm{f}\left(\tau\left(\mathrm{x}_{1}\right), \tau\left(\mathrm{x}_{2}\right), \ldots, \tau\left(\mathrm{x}_{\mathrm{n}}\right)\right) \forall \mathrm{f}\left(\mathrm{x}_{1}, \mathrm{x}_{2}, \ldots, \mathrm{x}_{\mathrm{n}}\right) \in \mathbf{K}\left[\mathrm{x}_{1}, \mathrm{x}_{2}, \ldots, \mathrm{x}_{\mathrm{n}}\right]$. Then $\mathbf{K S}_{\mathbf{n}}$ forms a group algebra with respect to addition of functions, product of functions by scalars and composition of functions which is called the group algebra of the symmetric group $S_{n}[3]$.

\section{Definition 2.2:}

Let $\mathrm{n}$ be a positive integer then the sequence $\lambda=\left(\lambda_{1}, \lambda_{2}, \ldots, \lambda_{1}\right)$ is called a partition of $\mathrm{n}$ if $\lambda_{1} \geq \lambda_{2} \geq \cdots \geq \lambda_{1}>0$ and $\lambda_{1}+$ $\lambda_{2}+\cdots+\lambda_{1}=n$. Then the set $D_{\lambda}=\left\{(\mathrm{i}, \mathrm{j}) \mid \mathrm{i}=1,2, \ldots, \mathrm{l} ; 1 \leq \mathrm{j} \leq \lambda_{\mathrm{i}}\right\}$ is called $\lambda$-diagram .And any bijective function $\mathrm{t}: \mathrm{D}_{\lambda} \rightarrow$ $\left\{\mathrm{x}_{1}, \mathrm{x}_{2}, \ldots, \mathrm{x}_{\mathrm{n}}\right\}$ is called a $\lambda$-tableau. $\mathrm{A} \lambda$-tableau may be thought as an array consisting of $\mathrm{l}$ rows and $\lambda_{1}$ columns of distinct 
variables $t((i, j))$ where the variables occur in the first $\lambda_{i}$ positions of the $i^{\text {th }}$ row and each variable $t((i, j))$ occurs in the $i^{\text {th }}$ row and the $j^{\text {th }}$ column $\left((i, j)\right.$-position)of the array.t((i,j)) will be denoted by $t(i, j)$ for each $(i, j) \in D_{\lambda}$.

The set of all $\lambda$-tableaux will be denoted by $T_{\lambda}$. i.e $T_{\lambda}=\{t \mid t$ is a $\lambda-$ tableau $\}$.

Then the function $\mathrm{g}: \mathrm{T}_{\lambda} \rightarrow \mathrm{K}\left[\mathrm{x}_{1}, \mathrm{x}_{2}, \ldots, \mathrm{x}_{\mathrm{n}}\right]$ which is defined by $\mathrm{g}(\mathrm{t})=\prod_{\mathrm{i}=1}^{\mathrm{l}} \prod_{\mathrm{j}=1}^{\lambda_{\mathrm{i}}}(\mathrm{t}(\mathrm{i}, \mathrm{j}))^{\mathrm{i}-1}, \forall \mathrm{t} \in \mathrm{T}_{\lambda}$. is called the row position monomial function of $T_{\lambda}$, and for each $\lambda$-tableau $t, g(t)$ is called the row position monomial of $t$.So $M(\lambda)$ is the cyclic $\mathrm{KS}_{\mathrm{n}}$-module generated by $\mathrm{g}(\mathrm{t})$ over $\mathrm{KS}_{\mathrm{n}} \cdot[2]$

\section{THE FIRST TRIANGULAR REPRESENTATIOM OF $\mathbf{S}_{\mathbf{n}}$}

In the beginning we define some denotations which we need them in this paper.

1) $\operatorname{Let}_{1}(\mathrm{n})=\sum_{i=1}^{n} \mathrm{x}_{\mathrm{i}}$.

2) Let $\sigma_{2}(\mathrm{n})=\sum_{1 \leq i<j \leq n} \mathrm{x}_{\mathrm{i}} \mathrm{x}_{\mathrm{j}}$

3) $\operatorname{LetC}_{l}(n)=x_{1}^{2}\left(\sigma_{2}(n)-\sum_{\substack{j=1 \\ j \neq l}}^{n} x_{l} x_{j}\right) ; l=1,2, \ldots, n$.

We denote $\overline{\mathrm{N}}$ to be the $\mathrm{KS}_{n}$ module generated by $C_{1}(\mathrm{n})$ over $\mathrm{KS}_{n}$. The set $B=\left\{\mathrm{C}_{\mathrm{i}}(\mathrm{n}) \mid \mathrm{i}=1,2, \ldots, \mathrm{n}\right\}$ is a $\mathrm{K}$-basis for $\overline{\mathrm{N}}=\mathrm{KS}_{\mathrm{n}} \mathrm{C}_{1}(\mathrm{n})$ and $\operatorname{dim}_{\mathrm{K}} \overline{\mathrm{N}}=\mathrm{n}$.

4) $\operatorname{Letu}_{i j}(n)=C_{i}(n)-C_{j}(n) ; i, j=1,2, \ldots, n$.

we denote $\overline{\mathrm{N}}_{0}$ the $\mathrm{KS}_{\mathrm{n}}$ submodule of $\overline{\mathrm{N}}$ generated by $\mathrm{u}_{12}(\mathrm{n})$.

5) Let $\sigma_{3}(\mathrm{n})=\sum_{1 \leq i<j \leq n} \sum_{\substack{k=1 \\ k \neq i, j}}^{n} \mathrm{x}_{\mathrm{i}} \mathrm{x}_{\mathrm{j}} \mathrm{x}_{\mathrm{k}}^{2}$.

Then $\sum_{l=1}^{n} C_{l}(n)=\sigma_{3}(n)$ and $\operatorname{dim}_{K}\left(K \sigma_{1}(n)\right)=\operatorname{dim}_{K}\left(K \sigma_{2}(n)\right)=\operatorname{dim}_{K}\left(K \sigma_{3}(n)\right)=1$.

$\mathrm{K} \sigma_{1}(\mathrm{n}), \mathrm{K} \sigma_{2}(\mathrm{n}) \operatorname{andK}_{3}(\mathrm{n})$ are all $\mathrm{KS}_{\mathrm{n}}$-modules, since $\tau \sigma_{\mathrm{k}}(\mathrm{n})=\sigma_{\mathrm{k}}(\mathrm{n}) \forall \mathrm{k}=1,2,3$.

\section{Definition 3.1:}

The $\mathrm{KS}_{\mathrm{n}}$-module $\mathrm{M}\left(\mathrm{n}-\frac{(\mathrm{r}+2)(\mathrm{r}+1)}{2}, \mathrm{r}+1, \mathrm{r}, \ldots, 1\right)$ defined by

$$
M\left(n-\frac{(r+2)(r+1)}{2}, r+1, r, \ldots, 1\right)=K_{n} x_{1} x_{2} \ldots x_{r+1} x_{r+2}^{2} \ldots x_{2 r+1}^{2} x_{2 r+2}^{3} \ldots x_{n}^{r+1}
$$

is called the $r^{\text {th }}$ triangular representation module of $S_{n}$ over $K$, where $n \geq \frac{(r+3)(r+2)}{2}$.

\section{Remark 3.1.1:}

The first triangular representation module of $S_{n}$ over $K$ is theKS $S_{n}$-module $M(n-3,2,1)$, the second triangular representation module of $S_{n}$ over $K$ is theKS $S_{n}-\operatorname{moduleM}(n-6,3,2,1)$, the third triangular representation module of $S_{n}$ over $\mathrm{K}$ is theKS $\mathrm{n}-\operatorname{moduleM}(\mathrm{n}-10,4,3,2,1)$, and so on.

\section{Lemma 3.2:}

The set $B(n-3,2,1)=\left\{x_{i} x_{j} x_{l}^{2} \mid 1 \leq i<j \leq n, 1 \leq l \leq n, l \neq i, j\right\} \quad$ is a K-basis of $M(n-3,2,1)$, anddim $m_{K} M(n-3,2,1)=$ $\left(\begin{array}{l}\mathrm{n} \\ 2\end{array}\right)(\mathrm{n}-2) ; \mathrm{n} \geq 6$.

\section{Theorem 3.3:}

The set $B_{0}(n-3,2,1)=\left\{x_{i} x_{j} x_{l}^{2}-x_{1} x_{2} x_{3}^{2} \mid 1 \leq i<j \leq n, 1 \leq l \leq n, l \neq i, j,(i, j, l) \neq(1,2,3)\right\}$ is a K-basis of $M_{0}(n-3,2,1)$, and $\operatorname{dim}_{K} M_{0}(n-3,2,1)=\left(\begin{array}{l}n \\ 2\end{array}\right)(n-2)-1 ; n \geq 6$.(see [5])

Theorem 3.4.: The set $B=\left\{C_{i}(n) \mid i=1,2, \ldots, n\right\}$ is a K- basis for $\bar{N}(n)=K_{n} C_{1}(n)$.

Proof:Let $\tau_{\mathrm{i}}=\left(\mathrm{x}_{1} \mathrm{x}_{\mathrm{i}}\right) \in \mathrm{S}_{\mathrm{n}} ; \mathrm{i}=2,3, \ldots, \mathrm{n}$. Then we get $\tau_{\mathrm{i}}\left(\mathrm{C}_{1}(\mathrm{n})\right)=\mathrm{C}_{\mathrm{i}}(\mathrm{n}) ; \mathrm{i}=2,3, \ldots, \mathrm{n}$

which implies that $C_{i}(n) \in \bar{N}(n)=K_{n} C_{1}(n)$ for all $i=1,2,3, \ldots, n$. Thus

$B=\left\{C_{i}(n) \mid i=1,2, \ldots, n\right\} \subseteq \bar{N}(n)$. Now if $\omega \in \bar{N}(n) \Rightarrow \omega \in K S_{n} C_{1}(n)$. 
$\Rightarrow \omega=\sum_{j=1}^{(n-1) !} \sum_{i=1}^{n} k_{j i} \sigma_{j i} C_{1}(n)$ where $\sigma_{j i} \in S_{n}$ and $\sigma_{j i}\left(x_{1}\right)=x_{i}\left(\right.$ i.e. $\left.\sigma_{j i}\left(C_{1}(n)\right)=C_{i}(n) \forall i=1,2, \ldots, n\right)$.

$\Rightarrow \omega=\sum_{i=1}^{n} \sum_{j=1}^{(n-1) !} k_{j i} \sigma_{j i} C_{1}(n)=\sum_{i=1}^{n}\left(\sum_{j=1}^{(n-1) !} k_{j i}\right) C_{i}(n)=\sum_{i=1}^{n} d_{i} C_{i}(n)$ whered $_{i}=\sum_{j=1}^{(n-1) !} k_{j i}$.Hence B generates $\bar{N}(n)$ over K .If

$\sum_{i=1}^{n} k_{i} C_{i}(n)=0$.i.e.

$k_{1} C_{1}(n)+k_{2} C_{2}(n)+\cdots+k_{n} C_{n}(n)=0$.which implies that

$k_{1}=k_{2}=\cdots=k_{n}=0 \operatorname{since} C_{l}(n)=\sum_{\substack{1 \leq i<j \leq n \\ l \neq i, j}} x_{i} x_{j} x_{l}^{2}$.Thus B is linearly independent. Therefore $\mathrm{B}$ is a basis of $\bar{N}(n)$ and $\operatorname{dim}_{K} \bar{N}(n)=n$.

Theorem 3.5.: $\bar{N}=K S_{n} C_{1}(n)$ and $M(n-1,1)$ are isomorphic over $K S_{n}$ (see[5])

Theorem 3.6.: $\overline{\mathrm{N}}_{0}=K S_{n} u_{12}(n)$ and $M_{0}(n-1,1)$ are isomorphic over $K S_{n}$.(see[5])

Corollary 3.6.1: The $K S_{n}$-module $\bar{N}_{0}=K S_{n} u_{12}(n)$ is irreducible over $K S_{n}$.(see [5])

Proposition 3.6.2: $\bar{N}=\bar{N}_{0} \oplus K \sigma_{3}(n)$.(see[5])

Proposition 3.6.3: $\bar{N}$ has the following two composition series

$0 \subset \bar{N}_{0} \subset \bar{N}$ and $0 \subset K \sigma_{3}(n) \subset \bar{N}$. (see [5])

\section{Definitions 3.7.:}

1. the $K S_{n}$-homomorphismd $: M(n-3,2,1) \rightarrow M(n-2,2)$ is defined in terms of the partial operators by

$$
d\left(x_{i} x_{j} x_{l}^{2}\right)=\sum_{k=1}^{n} \frac{\partial^{2}}{\partial x_{k}^{2}}\left(x_{i} x_{j} x_{l}^{2}\right),
$$

2. the $K S_{n}$-homomorphism $\bar{d}$ which is the restriction of $d$ to $M_{0}(n-3,2,1)$. i.e.

$$
\bar{d}: M_{0}(n-3,2,1) . \rightarrow M_{0}(n-3,2) .
$$

3. the $K S_{n}$-homomorphism $f: M(n-3,2,1) \rightarrow K$ which is defined by

$$
f\left(\sum_{1 \leq i<j \leq n} \sum_{\substack{l=1 \\ l \neq i, j}}^{n} k_{i, j, l} x_{i} x_{j} x_{l}^{2}\right)=\sum_{1 \leq i<j \leq n} \sum_{\substack{l=1 \\ l \neq i, j}}^{n} k_{i, j, l} .
$$

Theorem 3.8.: The following sequence of $K S_{n}$ - modules is exact

$0 \rightarrow \operatorname{Ker} d \stackrel{i}{\rightarrow} M(n-3,2,1) \stackrel{d}{\rightarrow} M(n-2,2) \rightarrow 0$

Corollary 3.8.1: The dimension of kerd over $K$ of the $K S_{n}-$ homomorphism

$d: M(n-3.2 .1) \rightarrow M(n-2,2)$ is $\frac{n(n-1)(n-3)}{2}$. (see [5])

Corollary 3.8.2: The following sequence of $K S_{n}-$ modules is exact

$0 \rightarrow \operatorname{Kerd} \stackrel{i}{\rightarrow} M_{0}(n-3,2,1) \stackrel{\bar{d}}{\rightarrow} M_{O}(n-2,2) \rightarrow 0$

Lemma 3.9.: $\operatorname{dim}_{K} S(n-3,2,1)=\frac{n(n-2)(n-4)}{3}$.

Proposition 3.10.: $S(n-3,2,1)$ is a proper submodule of kerd .(see [5])

Theorem 3.11.:The following sequence over the field $K$ is exact.

$O \rightarrow M_{0}(n-3,2,1) \stackrel{i}{\rightarrow} M(n-3,2,1) \stackrel{f}{\rightarrow} K \rightarrow O$ $\ldots(3)(\operatorname{see}[5])$

Corollary 3.11.1: the exact sequence (3) is split iff $p$ does not divide $\frac{n(n-1)(n-2)}{2}$

Proof: Assume that $p$ does not divide $\frac{n(n-1)(n-2)}{2}$. Define $g: K \rightarrow M(n-3,2,1)$ by 
$g(1)=\frac{2 \sigma_{3}(n)}{n(n-1)(n-2)}$ where $\sigma_{3}(n)=\sum_{1<i<j \leq n} \sum_{\substack{l=1 \\ l \neq i, j}}^{n} x_{i} x_{j} x_{l}^{2}$

Then $g$ is a $K S_{n}$-homomorphism since $\tau \sigma_{3}(n)=\sigma_{3}(n)$ for any $\tau \in S_{n}$ and $\tau(1)=1$, thus we get

$g(\tau 1)=g(1)=\frac{2 \sigma_{3}(n)}{n(n-1)(n-2)}=\tau\left(\frac{2 \sigma_{3}(n)}{n(n-1)(n-2)}\right)=\tau g(1)$.

Moreover we have $f g(1)=f(g(1))=f\left(\frac{2 \sigma_{3}(n)}{n(n-1)(n-2)}\right)=\frac{2}{n(n-1)(n-2)} f\left(\sigma_{3}(n)\right)$

$=\frac{2}{n(n-1)(n-2)} \cdot \frac{n(n-1)(n-2)}{2}=1$. Hence $f g=I$.Therefore the sequence (3) is split.

Now assume the sequence (3) is split .Hence there is a $K S_{n}$-homomorphism

$g: K \rightarrow M(n-3,2,1)$ s.t. $f g=I$. Then $g$ has the form

$g(1)=\sum_{1<i<j \leq n} \sum_{\substack{l=1 \\ l \neq i, j}}^{n} k_{i j l} x_{i} x_{j} x_{l}^{2}$.

Then for any $\tau=\left(x_{r} x_{s}\right) \in S_{n} ; 1 \leq r<s \leq n$ we have $g(1)=g(\tau 1)$.

Thus we get $0=g(1)-g(\tau 1)$ i. e .

$$
\begin{aligned}
& 0=\sum_{1<i<j \leq n} \sum_{\substack{l=1 \\
l \neq i, j}}^{n} k_{i j l} x_{i} x_{j} x_{l}^{2}-\sum_{1<i<j \leq n} \sum_{\substack{l=1 \\
l \neq i, j}}^{n} k_{i j l} \tau x_{i} x_{j} x_{l}^{2}=\sum_{\substack { 1 \leq i<j \leq n \\
\begin{subarray}{c}{l=1 \\
l \neq i, j{ 1 \leq i < j \leq n \\
\begin{subarray} { c } { l = 1 \\
l \neq i , j } }\end{subarray}}^{n} k_{\mathrm{i} j l}\left(x_{i} x_{j} x_{l}^{2}-\tau\left(x_{i} x_{j} x_{l}^{2}\right)\right) \\
& =\sum_{\substack{j=r+1 \\
j \neq s}}^{n} \sum_{\substack{l=1 \\
l \neq r, s, j}}^{n}\left(k_{r j l}-k_{s j l}\right) x_{r} x_{j} x_{l}^{2}+\sum_{j=s+1}^{n} \sum_{\substack{l=1 \\
l \neq r, s, j}}^{n}\left(k_{s j l}-k_{r j l}\right) x_{s} x_{j} x_{l}^{2}+\sum_{i=1}^{r-1} \sum_{\substack{l=1 \\
l \neq r, s, i}}^{n}\left(k_{i r l}-k_{i s l}\right) x_{i} x_{r} \\
& +\sum_{\substack{i=1 \\
i \neq r}}^{s-1} \sum_{\substack{l=1 \\
l \neq r, s, i}}^{n}\left(k_{i s l}-k_{i r l}\right) x_{i} x_{s} x_{l}^{2}+\sum_{\substack{i=1 \\
i \neq r, s}}^{n-1} \sum_{\substack{j=i+1 \\
j \neq r, s}}^{n}\left(k_{i j r}-k_{i j s}\right) x_{i} x_{j} x_{r}^{2}+\sum_{\substack{i=1 \\
i \neq r, s}}^{n-1} \sum_{\substack{j=i+1 \\
j \neq r, s}}^{n}\left(k_{i j s}-k_{i j r}\right) x_{i} x_{j} x_{s}^{2} \\
& +\sum_{\substack{j \neq r+1 \\
j \neq s}}^{n}\left(k_{r j s}-k_{s j r}\right) x_{r} x_{j} x_{s}^{2}+\sum_{j=s+1}^{n}\left(k_{s j r}-k_{r j s}\right) x_{s} x_{j} x_{r}^{2}+\sum_{i=1}^{r-1}\left(k_{i r s}-k_{i s r}\right) x_{i} x_{r} x_{s}^{2}+\sum_{\substack{i=1 \\
r \neq i}}^{s-1}\left(k_{i s r}-k_{i r s}\right) x_{i} x_{s} x_{r}^{2} \\
& =\sum_{\substack{j=r+1 \\
j \neq s}}^{n} \sum_{\substack{l=1 \\
l \neq r, s}}^{n}\left(k_{r j l}-k_{s j l}\right)\left(x_{r} x_{j} x_{l}^{2}-x_{s} x_{j} x_{l}^{2}\right) \\
& +\sum_{i=1}^{r-1} \sum_{\substack{l=1 \\
l \neq r, s}}^{n}\left(k_{i r l}-k_{i s l}\right)\left(x_{i} x_{r} x_{l}^{2}-x_{i} x_{s} x_{l}^{2}\right)+\sum_{\substack{i=1 \\
i \neq r, s}}^{n-1} \sum_{\substack{j=i+1 \\
j \neq r, s}}^{n}\left(k_{i j r}-k_{i j s}\right)\left(x_{i} x_{j} x_{r}^{2}-x_{i} x_{j} x_{s}^{2}\right) \\
& +\sum_{\substack{r<j \leq n \\
j \neq s}}\left(k_{r j s}-k_{s j \mathrm{r}}\right)\left(x_{r} x_{j} x_{s}^{2}-x_{s} x_{j} x_{r}^{2}\right)+\sum_{1 \leq i<r<n}\left(k_{i r s}-k_{i s r}\right)\left(x_{i} x_{r} x_{s}^{2}-x_{i} x_{s} x_{r}^{2}\right) .
\end{aligned}
$$

Which implies by equating the coefficient of the above equation that

$k_{r j l}=k_{s j l} \forall r \leq j<n \ni j \neq s$ and $\forall 1 \leq l \leq n \ni l \neq r, s, j$.

$k_{\text {irl }}=k_{\text {isl }} \quad \forall 1 \leq i<r$ and $\forall 1 \leq l \leq n \ni l \neq r, s, j$.

$k_{i j r}=k_{i j s} \quad \forall 1 \leq i<j<n \ni i, j \neq r, s$.

$k_{\text {irs }}=k_{i s r} \quad \forall 1 \leq i<r$

$$
k_{r j s}=k_{s j r} \quad \forall r<j \leq n, j \neq s .
$$

Hence for each $r$, s s.t $1 \leq r<s \leq n$ we get $k_{i j l}=k ; 1 \leq i<j \leq n$

and $1 \leq l \leq n \ni l \neq i, j$. Thus

$g(1)=\sum_{1\langle i<j \leq n} \sum_{\substack{l=1 \\ l \neq i, j}}^{n} k_{i j l} x_{i} x_{j} x_{l}^{2}=\sum_{1<i<j \leq n} \sum_{\substack{l=1 \\ l \neq i, j}}^{n} k x_{i} x_{j} x_{l}^{2}=k \sum_{1\langle i<j \leq n} \sum_{\substack{l=1 \\ l \neq i, j}}^{n} x_{i} x_{j} x_{l}^{2}=k \sigma_{3}(n)$. 
$\because f g=I$. Hence $1=f g(1)=f\left(k \sigma_{3}(n)\right)=k f\left(\sigma_{3}(n)\right)=\frac{k}{2} n(\mathrm{n}-1)(n-2)$

Thus $p$ does not divide $\frac{n(n-1)(n-2)}{2}$.

Corollary 3.11.2: $M_{0}(n-3,2,1)$ is not a direct summand of $M(n-3,2,1)$ when $p$ divides $\frac{n(n-1)(n-2)}{2}$.

Proof: Assume that $M_{0}(n-3,2,1)$ is a direct summand of $M(n-3,2,1)$ when $p$ divides

$\frac{n(n-1)(n-2)}{2}$. Hence there is a $K S_{n}$ submodule of $M(n-3,2,1)$, say $H$, s.t. $M(n-3,2,1)=$

$M_{0}(\mathrm{n}-3,2,1) \oplus H$. Thus the exact sequence (3) is split and this is a contradicts corollary(3.11.1).

Therefore $M_{0}(\mathrm{n}-3,2,1)$ is not a direct summand of $M(n-3,2,1)$.

Proposition 3.12: If $p$ divides $2(n-2)$, then $K \sigma_{3}(n) \subset$ ker $d$.

Proof : By the definition of $\sigma_{3}(n)$ we have that

$\sigma_{3}(n)=\sum_{1 \leq i j \leq n} \sum_{\substack{l=1 \\ l \neq i, j}}^{n} x_{i} x_{j} x_{l}^{2}$. Thus $d\left(\sigma_{3}(n)\right)=2(n-2) \sum_{1 \leq i, j \leq n} x_{i} x_{j}=2(n-2) \sigma_{2}(n) \quad$.Which implies that $K \sigma_{3}(n) \subset$ ker $d$ when $p$ divides $2(n-2)$.

Theorem3.13: If $p \neq 2$ and $p$ divides $(n-2)$ then we have the following series:

1) $0 \subset K \sigma_{3} \subset \operatorname{ker} d \subset \bar{N}_{0} \oplus$ ker $d \subset M_{0}(n-3,2,1) \subset M(n-3,2,1)$

2) $0 \subset \bar{N}_{0} \subset \bar{N} \subset \bar{N}_{0} \oplus$ ker $d \subset M_{0}(n-3,2,1) \subset M(n-3,2,1)$.

3) $0 \subset K \sigma_{3} \subset \bar{N} \subset \bar{N}_{0} \oplus$ ker $d \subset M_{0}(n-3,2,1) \subset M(n-3,2,1)$.

\section{Proof :}

Since $p \neq 2$ and $p$ divides $(n-2)$, then by proposition (3.12) we get that $K \sigma_{3}(n) \subset k e r d$.

Moreover when $p$ divides $(n-2)$ implies $p$ does not divides $n$. Thus we get that $\bar{N}_{0}$ is irreducible

submodule overKS $S_{n}$ by corollary (3.6.1). Hence $\bar{N}_{0} \oplus$ ker $d \subset M_{0}(n-3,2,1)$.

Therefore we get the following series

1) $0 \subset K \sigma_{3} \subset \operatorname{ker} \mathrm{d} \subset \overline{\mathrm{N}}_{0} \oplus \operatorname{ker} \mathrm{d} \subset \mathrm{M}_{0}(\mathrm{n}-3,2,1) \subset \mathrm{M}(\mathrm{n}-3,2,1)$

2) $0 \subset \overline{\mathrm{N}}_{0} \subset \overline{\mathrm{N}} \subset \overline{\mathrm{N}}_{0} \oplus \operatorname{kerd} \subset \mathrm{M}_{0}(\mathrm{n}-3,2,1) \subset \mathrm{M}(\mathrm{n}-3,2,1)$.

3) $0 \subset \mathrm{K} \sigma_{3} \subset \overline{\mathrm{N}} \subset \overline{\mathrm{N}}_{0} \oplus$ ker $\mathrm{d} \subset \mathrm{M}_{0}(\mathrm{n}-3,2,1) \subset \mathrm{M}(\mathrm{n}-3,2,1)$.

Theorem 3.14: The following sequence of a $\mathrm{KS}_{\mathrm{n}}$-submodule is exact.

$0 \rightarrow \operatorname{kerd}_{1} \stackrel{\mathrm{i}}{\rightarrow} \mathrm{T} \stackrel{\mathrm{d}_{1}}{\rightarrow} \mathrm{S}(\mathrm{n}-2,2) \rightarrow 0 \quad(4)$

whereT $=\mathrm{KS}_{\mathrm{n}}\left(\mathrm{x}_{1} \mathrm{x}_{3} \mathrm{x}_{5}^{2}-\mathrm{x}_{1} \mathrm{x}_{4} \mathrm{x}_{5}^{2}+\mathrm{x}_{2} \mathrm{x}_{4} \mathrm{x}_{5}^{2}-\mathrm{x}_{2} \mathrm{x}_{3} \mathrm{x}_{5}^{2}\right)$.

Proof: The same proof of theorem(3.13) in [6].

Corollary 3.14.1: The exact sequence (4) over the field $\mathrm{K}$ is split.

Proof : As the proof of corollary(3.13.1) in[6].

Proposition 3.15: $\mathrm{S}(\mathrm{n}-3,2,1)$ is a properKS $\mathrm{n}$-submodule of $\mathrm{T}$.

Proof: As the proof of proposition(3.13.2)in[6]. 


\section{References:}

1) Adalbert Kerber, Axel Kohnert, Modular irreducible representations of the Symmetric group as linear Codes ,European Journal of Combinatorics 25(2004) 1285-1299.

2)M.H .Peel, Specht Modules and Symmetric groups .Journal of Algebra.vol.36 No.1(1975)88-97).

3) M.H .Peel, On the Second Natural Representation of the Symmetric groups. Glasgow Math .Journal vol.10 part1(1969),25-37.

4) M.H .Peel, Hook Representation of the Symmetric groups ,Glasgow Math .Journal vol.12 part 2(1971),136-149.

5)EyadM.A.Al-Aamily,AliAbdulsahibM.Al-Butaihi,Abid Ali H.Al-Ta'ai,The First Triangular Representation of The Symmetric Groups with $p$ divides (n-1).IOSR JM Volume 12, Issue 2 Ver. II (Mar. - Apr. 2016), PP 27-34

6)EyadM.A.Al-Aamily,AliAbdulsahibM.Al-Butaihi,Abid Ali H.Al-Ta'ai,The First Triangular Representation of The Symmetric Groups over a field K of characteristic $p=0$,Journal of Advances in Mathematics vol.12 no.4(May-2016),6121-6126. 